\title{
Evaluación de materiales flotantes biodegradables para cultivos hidropónicos de lechuga (Lactuca sativa)
}

Evelin TAMayo GutiÉRreZ ${ }^{1}$ Bonye Carrera Pozo ${ }^{2}$ MAURICIO RACINES-OLIVA ${ }^{3}$

Recibido: 20/05/2018 Aceptado: 04/11/2019 Publicado: 16/10/2020

\begin{abstract}
RESUMEN
La presente investigación propone una alternativa de material biodegradable para uso en cultivos en medios hidropónicos de lechuga mediante el sistema de producción a raíz desnuda, con el objetivo de sustituir funcionalmente al poliestireno expandido, utilizado de manera habitual como material de soporte, a pesar de ser un elemento altamente contaminante. La propuesta tiene base en la elaboración de módulos individuales de material flotante a partir de desechos agroindustriales con un recubrimiento impermeabilizante de cera orgánica. Los materiales fueron elaborados en la ciudad de Quito, Ecuador, y las variables evaluadas fueron: dureza de superficies, resistencia a la compresión y a la flexión, y contaminación microbiológica del material durante el tiempo de cultivo del vegetal. Como resultado, se obtuvo que la propuesta con mayor adaptabilidad al cultivo fue la elaborada a partir de fibra de palma (Elaeis guineensis) aglutinada con arroz en proporción de $70 \%$ $30 \%$, respectivamente, con adición de cera de carnauba como cobertura superficial.
\end{abstract}

Palabras clave: material flotante; cultivo hidropónico; poliestireno expandido; fibra de palma.

\section{INTRODUCCIÓN}

El cultivo hidropónico de hortalizas constituye una alternativa de cultivo intensiva, de la cual se obtienen altos volúmenes de producción por metro cuadrado y que se puede adaptar a lugares en donde el suelo no es apto para la agricultura, en espacios limitados de terreno o en áreas donde el recurso hídrico sea limitado, ya que el sistema de recirculación de agua permite el ahorro de hasta el $90 \%$ del líquido vital frente al cultivo de vegetales en suelo (Cuá y Vázquez, 2016).

Los cultivos hidropónicos utilizan materiales que sustituyen a la tierra y que, además, brindan un entorno favorable para la planta, permitiendo su crecimiento normal. Para el cultivo hidropónico a raíz desnuda, la producción se desarrolla de manera que la planta queda fija en un material flotante de soporte sobre la solución nutritiva acuosa durante todo el ciclo de cultivo, hasta alcanzar las características aptas para su cosecha (Guzmán, 2004).

El presente trabajo aborda el sistema de cultivo hidropónico a raíz desnuda de lechuga, para el cual se utilizan, usualmente, como base y soporte de las plantas, planchas de poliestireno expandido.

El poliestireno expandido, por su parte, al ser un material estable, de baja densidad y de fácil acceso para ser utilizado en este método de cultivo, luego de su vida útil, constituye uno de los materiales más contaminantes debido a su escasa capacidad de biodegradabilidad (Rodríguez, 14 de setiembre de 2013). Esto significa un problema ambiental de gran impacto, sobre el cual se están reuniendo esfuerzos para poder encontrar un material amigable con el ambiente que pueda sustituir al mencionado poliestireno (Tamayo, Sarasty y Mosquera, 2017).

Doctora en Ingeniería Industrial por la Universidad Nacional Mayor de San Marcos (Lima, Perú). Actualmente, es docente de Ingeniería Agroindustrial y de Alimentos en la Facultad de Ingenierías y Ciencias Aplicadas de la Universidad de Las Américas. (Quito, Ecuador). ORCID: https://orcid.org/0000-0003-3200-8229

E-mail: evelin.tamayo@udla.edu.ec

2 Ingeniera agroindustrial y de alimentos por la Universidad de Las Américas (Quito, Ecuador). Actualmente, es consultora independiente. (Quito, Ecuador).

ORCID: https://orcid.org/0000-0002-5740-0748

E-mail: bmcarrera@udlanet.ec

$3 \mathrm{Ph}$. D. en Bioscience Engineering por la KU Leuven (Lovaina, Bélgica). Actualmente, es docente investigador en Ingeniería Agroindustrial y de Alimentos de la Universidad de Las Américas. (Quito, Ecuador)

ORCID: https://orcid.org/0000-0003-4335-4311

E-mail: mauricio.racines@udla.edu.ec 
En virtud de lo anterior, la propuesta tuvo como objetivo generar materiales flotantes biodegradables, fabricados a partir de desechos agroindustriales, como una alternativa al uso de poliestireno expandido, y que puedan cumplir las funciones de soporte de plantas para el cultivo hidropónico a raíz desnuda de lechuga.

Los insumos elegidos para la construcción de aglomerados de fibras vegetales, primeramente, fueron los desechos agroindustriales de banano (Musa paradisiaca) en razón de que existen estudios favorables sobre el uso de este tipo de fibra a nivel industrial, como el de Manrique y Rivera (2012). Además, se realizó la comparación de lo anterior con el comportamiento de la fibra presente en el fruto de la palma aceitera (Elaeis guineensis). Finalmente, se empleó el arroz (Oryza sativa) como material aglutinante por su propicia cantidad de almidón (López y Ozaeta, 2013). Para esta elección, se tomó en consideración que los cultivos mencionados son de importancia económica en Ecuador y que constituyen un suministro de materia prima de fácil acceso y bajo costo.

Para el caso de la fibra de palma aceitera, se estima que la cantidad que se obtiene, luego de la extracción del aceite, alcanza el $58.7 \%$ de su peso inicial (Bejarano, 2006). Por su lado, la fibra de banano fue extraída del raquis del racimo, el cual constituye entre el $12 \%$ y $15 \%$ del peso total del mismo. En el caso del arroz, se utilizó el subproducto denominado arrocillo, es decir, el arroz partido que se obtiene de las piladoras, este es un subproducto del proceso y representa un $10 \%$ del peso del arroz que ingresa al proceso de pilado (Benavidez, 2006).

Para elaborar los prototipos se utilizaron operaciones unitarias simplificadas, con el mínimo gasto energético y sin la emisión de gases tóxicos contaminantes, con la finalidad de que esta tecnología pueda estar al alcance de productores que deseen desarrollar sus propios materiales de soporte.

Por otra parte, es importante recalcar que la oportunidad de utilizar subproductos y desechos agroindustriales abre las puertas a nuevas fuentes de ingreso económico, pues se propicia la generación de alternativas de productos con valor agregado $y$, si es que estas propuestas son desarrolladas a nivel industrial, se producen fuentes de trabajo (Tapia, 2007).

\section{METODOLOGÍA}

\section{Material y métodos}

La presente investigación tuvo como localidad a la ciudad de Quito, Ecuador, situada a 2850 m s. n. m., con temperatura anual promedio de $13.9{ }^{\circ} \mathrm{C}$ y con $1273 \mathrm{~mm}$ de precipitación anual. La base técnica fue brindada por el diseño de aglomerados de fibras vegetales descrito en el estudio presentado por Tamayo et al. (2017). En el mencionado contexto, se utilizaron fibras de palma aceitera (Elaeis guineensis) y banano (Musa paradisiaca), aglutinadas con arrocillo de variedad INIAP15 y recubrimiento de ceras de origen orgánico: de abeja y de palma carnauba.

Por motivos de accesibilidad en la obtención de materiales, se utilizó la fórmula probada por el estudio indicado, la cual se basa en la fibra de banano al $70 \%$ y arrocillo al $30 \%$, ya que es un tratamiento con excelentes resultados en cuanto a aglutinación, compactación y homogeneidad; además, lo anterior se evaluó comparativamente frente a aglomerados a base de fibra de palma aceitera en iguales concentraciones.

En la Figura 1, se muestra el diagrama del proceso utilizado en la elaboración del prototipo:

Se aplicó en el experimento el diseño estadístico de bloques completamente al azar (DBCA) con un análisis de varianza (ANOVA), además de una prueba de Tukey $(p \leq 0.05)$ para comparación con el software SPSS (International Business Machines Corporation, EE. UU.).

Para la evaluación, se elaboraron 25 aglomerados de $12 \times 12 \times 3 \mathrm{~cm}$ de cada uno de los tratamientos que se describen en la Tabla 1.

En la Figura 2, se presenta el material aglomerado producido con fibras vegetales y cobertura de polímeros orgánicos, el cual fue utilizado para la evaluación en campo de cultivo de lechuga hidropónica.

Los prototipos desarrollados fueron expuestos a evaluaciones mecánicas de materiales: evaluación de la dureza de superficies y resistencia a la compresión y a la flexión, las mismas que se muestran en la Figura 3 (secciones A, B y C, respectivamente); además, los modelos fueron sometidos a evaluaciones microbiológicas.

Con respecto a las pruebas mecánicas, fueron evaluadas 6 repeticiones de cada tratamiento.

En la evaluación de dureza superficial, se hizo uso de un penetrómetro en escala Shore y se midió de manera manual la superficie de las dos caras del material. Para realizar la prueba, se contó con una plantilla en cruz con la finalidad de homologar la toma de muestra en todos los prototipos, con lo que se consiguió un total de 32 penetraciones por cara. 


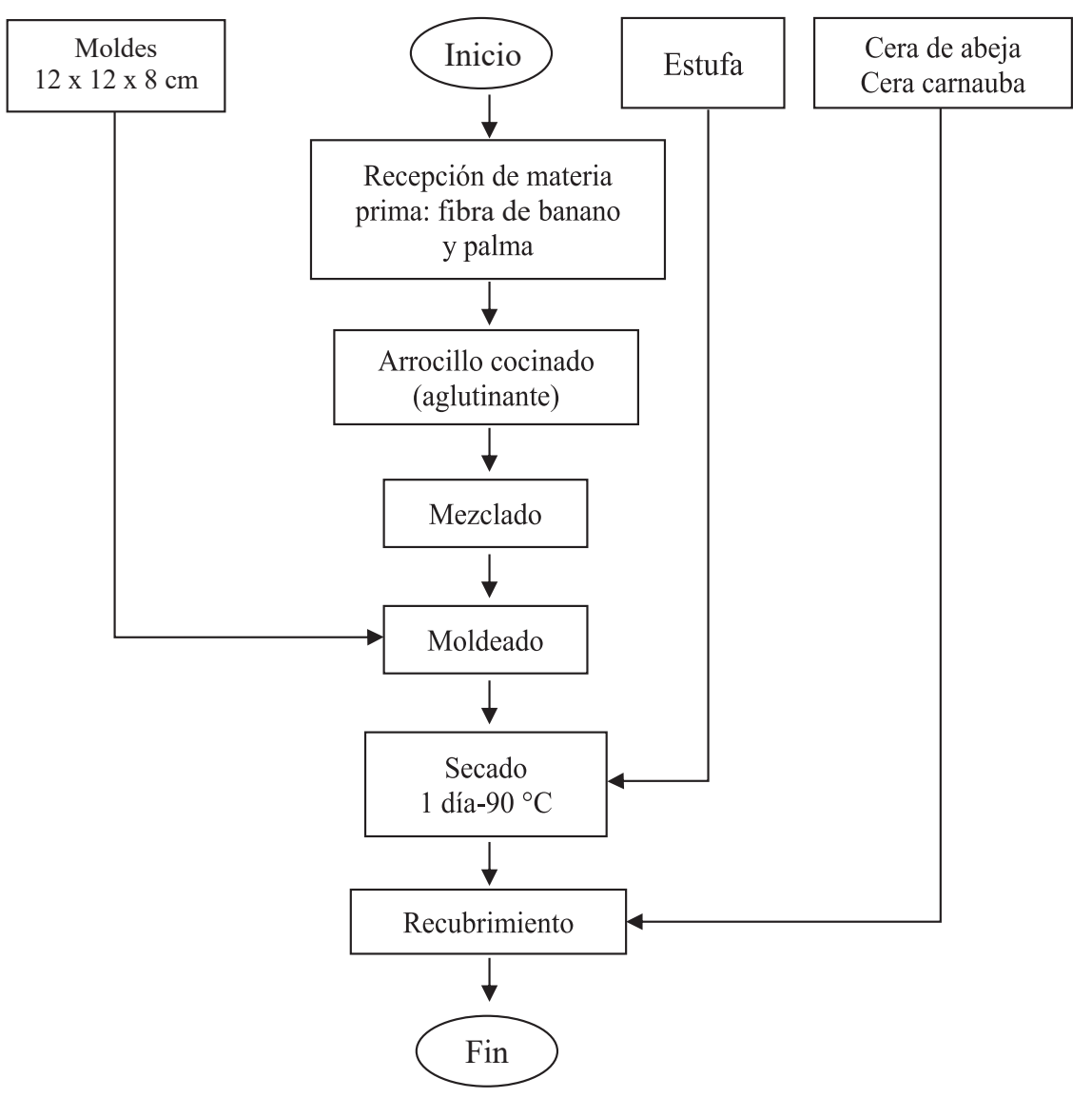

Figura 1. Diagrama de procesos para la construcción de los prototipos de materiales flotantes.

Fuente: Elaboración propia.

Tabla 1. Descripción de tratamientos.

\begin{tabular}{|c|l|c|c|l|}
\hline Tratamiento & Origen de la fibra & $\begin{array}{c}\text { Fibra en el aglomerado } \\
\text { (\% peso seco) }\end{array}$ & $\begin{array}{c}\text { Arrocillo en el aglomerado } \\
\text { (\% peso seco) }\end{array}$ & \multicolumn{1}{|c|}{ Recubrimiento } \\
\hline 1 & Banano & $70 \%$ & $30 \%$ & Cera carnauba \\
\hline 2 & Palma aceitera & $60 \%$ & $40 \%$ & Cera carnauba \\
\hline 3 & Banano & $50 \%$ & $50 \%$ & Cera de abeja \\
\hline 4 & Palma aceitera & $70 \%$ & $30 \%$ & Cera de abeja \\
\hline 5 & \multicolumn{2}{|l}{} \\
\hline
\end{tabular}

Fuente: Elaboración propia.

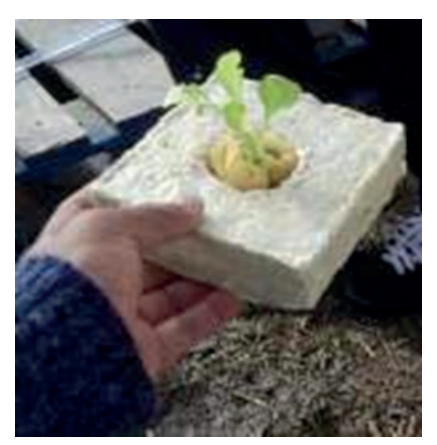

Figura 2. Prototipo de material aglomerado con cobertura de polímero orgánico.

Fuente: Elaboración propia. 
Para las pruebas mecánicas de resistencia a la flexión y la compresión, fueron utilizadas probetas de $12 \times 4 \times 3 \mathrm{~cm}$. La resistencia a la compresión fue medida con ayuda del equipo de compresión de 3000 kN presentado en la Figura 3, sección B; el dato proporcionado fue el de carga, medido en $\mathrm{kN}$. En la evaluación de la variable de resistencia a la flexión con carga transversal, se utilizó el equipo de ensayos Ilamado Universal (ver la Figura 3, sección C), el cual cuenta con una potencia de compresión de $200 \mathrm{kN}$, para obtener el punto de quiebre de cada muestra expuesta.
Se procedió a la evaluación microbiológica con la premisa de que el material desarrollado estaría en contacto con vegetales de consumo directo en crudo, es por esto que se consideró de importancia su valoración.

Para el fin descrito, fue construido un sistema de cultivo en módulos independientes, el mismo que se aprecia en la Figura 4, con 6 observaciones cada uno, mediante el cual se desarrolló el cultivo de lechuga en medio hidropónico y se valoró la contaminación microbiológica del material.

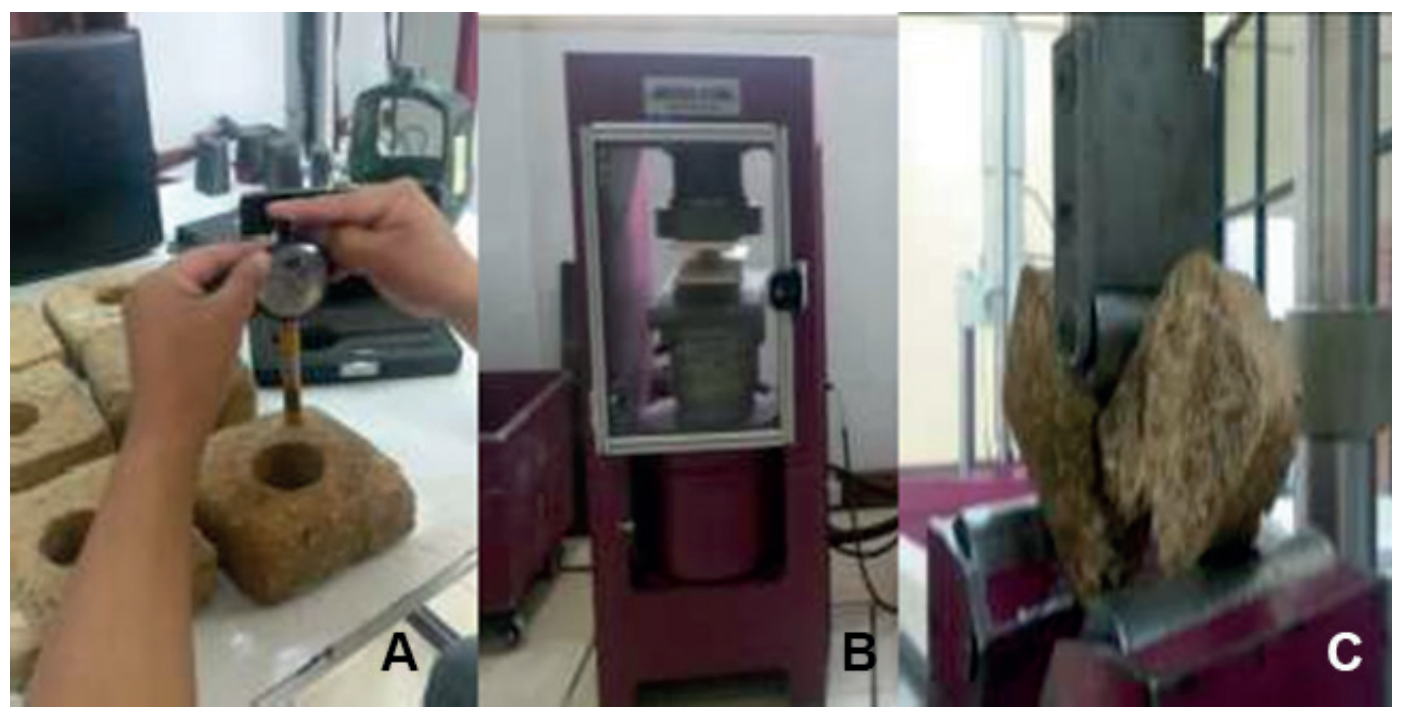

Figura 3. Evaluaciones mecánicas. A: medición de dureza con penetrómetro; B: medición de resistencia a la compresión; C: medición de resistencia a la flexión.

Fuente: Elaboración propia.

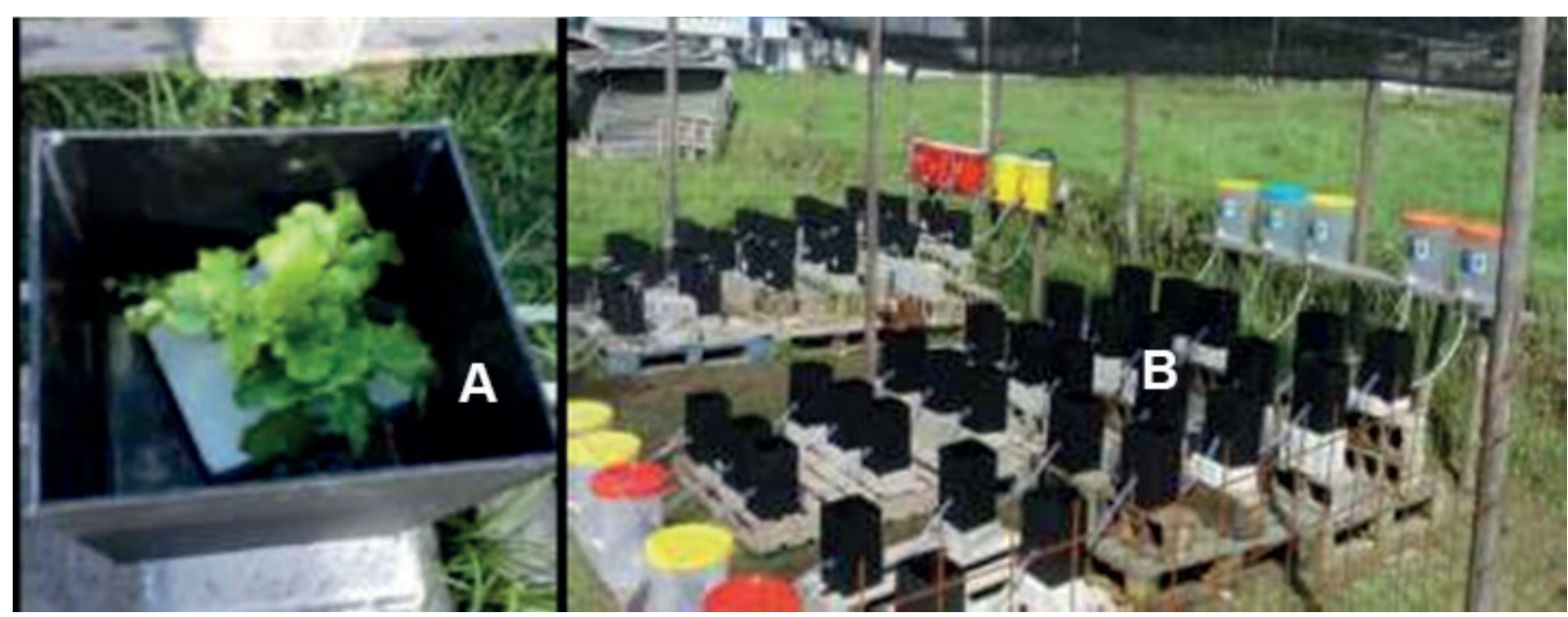

Figura 4. Sistema de cultivo. A: evaluación del material en sistema hidropónico a raíz flotante en módulo individual; B: módulos de recirculación para evaluación del cultivo hidropónico de lechuga.

Fuente: Elaboración propia. 
Se realizó la evaluación microbiológica mediante la toma de muestra en zigzag de la superficie de cada prototipo en evaluación. Asimismo, se consideraron dos etapas clave para la toma de muestras: a los 15 días luego de la siembra y a los 30 días, que corresponde al tiempo de cosecha de la lechuga.

Para la evaluación de contaminación microbiológica, se aplicó el uso de placas Compact Dry, las cuales favorecen el cultivo uniforme y estándar de las muestras. Algunas de las características de dichas placas son:

- Compact Dry TC: facilita la identificación de aerobios totales por medio de tinción con tetrazolio en un medio de agar, coloreando las colonias de rojo.

- Compact Dry YM: permite la observación de colonias de mohos y levaduras. La característica del sustrato es que posee el compuesto X-Phos, lo que permite la coloración de las colonias de azul.

Las placas utilizadas cuentan con el aval de las certificaciones AOAC, MicroVal y NordVal (Hyserve, 2010).

Los protocolos empleados fueron los siguientes:

\section{Métodos para la toma de muestras superficiales y el transporte}

- Utilización de guantes de látex para evitar contaminación cruzada.

- Toma de muestras al azar.

- Raspado en forma de zigzag con un hisopo estéril.
- Colocación de los hisopos en tubos de ensayo con agua destilada para impedir algún tipo de afección hacia los microorganismos.

- Transporte de los tubos de ensayo en condiciones seguras para evitar rupturas y cambios de temperatura que puedan afectar a la muestra.

\section{Siembra de muestras en las placas de conteo}

- Se realizaron diluciones de la muestra madre en $10 \mathrm{ml}$ de agua destilada y se colocaron en tubos de ensayo.

- Subsecuentemente, se realizaron disoluciones a la concentración $10^{-4}$.

- Se colocó un mililitro de la solución descrita en el centro de los Compact Dry, etiquetándolos de acuerdo al tratamiento.

- Las placas de Petri Compact Dry TC se incubaron a temperaturas de entre $35^{\circ} \mathrm{C}$ y $37^{\circ} \mathrm{C}$ por 24 horas.

- Las cajas de cultivo se incubaron a temperaturas de entre $25^{\circ} \mathrm{C}$ y $30{ }^{\circ} \mathrm{C}$ por 2 a 3 días.

Las placas de trabajo y el conteo de colonias se muestran en la Figura 5.

\section{RESULTADOS}

La metodología utilizada para la elaboración de prototipos a partir de fibras vegetales aglutinadas con arrocillo, descrita por Tamayo et al. (2017), generó materiales homogéneos y estables, aptos para su utilización en el sistema hidropónico estudiado.

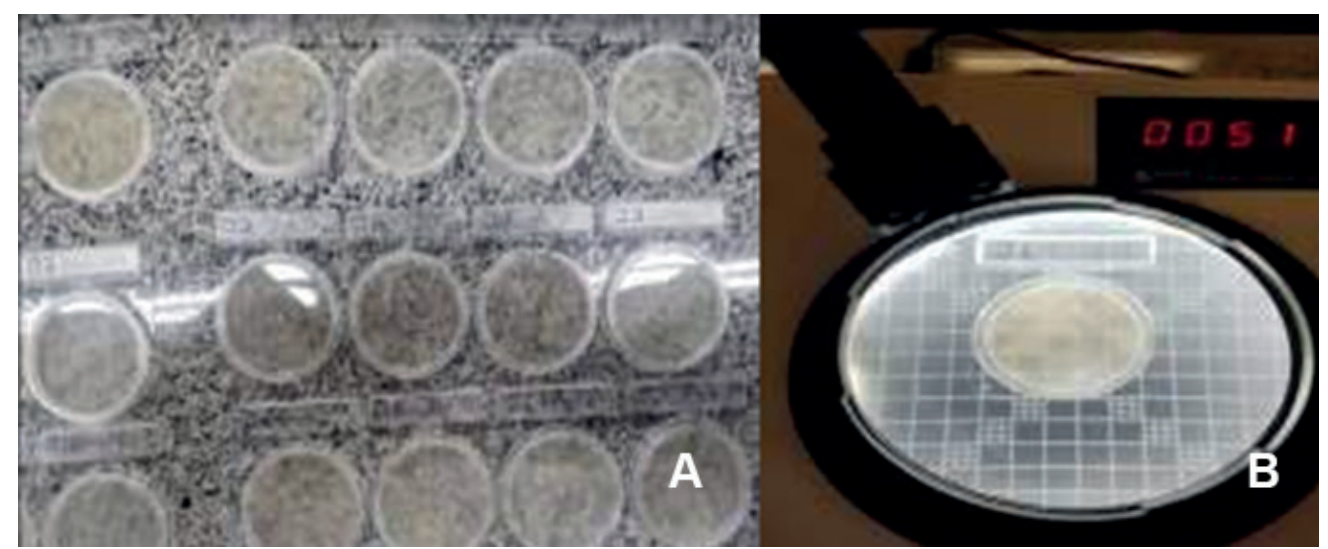

Figura 5. Evaluaciones microbiológicas. A: siembra de muestras en métodos de cultivo específicos; $\mathrm{B}$ : conteo de colonias.

Fuente: Elaboración propia. 
Los resultados de la valoración de dureza superficial presentaron diferencia significativa entre los tratamientos, de donde el prototipo constituido por $70 \%$ de palma, $30 \%$ de arrocillo y con cobertura de carnauba resultó ser el material con mayor dureza en comparación con sus homólogos.

Es importante indicar que no existe diferencia estadística entre los tratamientos 1, 3 y 4 , datos que se encuentran en la Tabla 2 a continuación.

\section{Estudio de resultados de la evaluación de resis- tencia a la compresión}

Los análisis de resultados de la varianza de los valores de carga arrojaron un $p$-valor menor a 0.05 , reflejo de que no presenta diferencia significativa entre las evaluaciones de los tratamientos; sin embargo, se pudo encontrar que sí existió diferencia entre los tratamientos y el testigo, donde este último fue mucho menor en resistencia a la compresión. En la Tabla 3, se exponen los promedios alcanzados (en kN) de los tratamientos en estudio.

\section{Evaluación de resultados de la prueba de resis- tencia a la flexión con carga transversal}

Los valores obtenidos proporcionan como resultado un $p$-valor mayor a 0.05 , lo que sugiere que los valores tomados en los tratamientos no difieren entre sí, es decir, que, con respecto a la resistencia a la flexión antes de ruptura, los prototipos en su totalidad presentan una resistencia similar, resultado muy superior a lo descrito por el testigo. En la Tabla 4 , se muestran los promedios en $\mathrm{kN}$ de la resistencia a la flexión, obtenidos mediante la evaluación de los prototipos.

\section{Análisis microbiológico de mesófilos aerobios a los 15 y 30 días}

Para la cuenta de unidades formadoras de colonias de mesófilos aerobios, se utilizó la metodología descrita. Los prototipos fueron sometidos a evaluación a los 15 días, estos resultados se exponen en la Tabla 5.

Tabla 2. Resultados de análisis de significancia de la evaluación en dureza mediante Tukey.

\begin{tabular}{|c|c|c|}
\hline Tratamiento & $\sigma \pm S^{2}$ & Grupo de significancia \\
\hline T1: banano $70 \%$, arrocillo $30 \%$, cobertura de cera carnauba & $17.87 \pm 0.35$ & $\mathrm{~b}$ \\
\hline T2: palma $70 \%$, arrocillo $30 \%$, cobertura de cera carnauba & $23.12 \pm 0.16$ & c \\
\hline T3: banano $70 \%$, arrocillo $30 \%$, cobertura de cera de abeja & $15.90 \pm 0.86$ & $\mathrm{~b}$ \\
\hline T4: palma $70 \%$, arrocillo $30 \%$, cobertura de cera de abeja & $16.73 \pm 0.11$ & $\mathrm{~b}$ \\
\hline
\end{tabular}

Fuente: Elaboración propia.

Tabla 3. Análisis de carga por prueba Tukey.

\begin{tabular}{|l|c|}
\hline \multicolumn{1}{|c|}{ Tratamiento } & Promedio (kN) \\
\hline T1: banano 70\%, arrocillo 30\%, cobertura de cera carnauba & $364.50 \pm 12.15$ \\
\hline T2: palma 70\%, arrocillo 30\%, cobertura de cera carnauba & $374.00 \pm 10.05$ \\
\hline T3: banano 70\%, arrocillo 30\%, cobertura de cera de abeja & $432.33 \pm 18.85$ \\
\hline T4: palma 70\%, arrocillo 30\%, cobertura de cera de abeja & $420.67 \pm 14.17$ \\
\hline Testigo: poliestireno expandido & $245 \mathrm{kN}$ \\
\hline
\end{tabular}

Fuente: Elaboración propia a partir de algunos datos tomados de Asociación Nacional de Poliestireno Expandido (2011).

Tabla 4. Resultados de la evaluación en resistencia a la flexión con carga transversal por prueba Tukey.

\begin{tabular}{|l|c|}
\hline \multicolumn{1}{|c|}{ Tratamiento } & Promedio (kN) \\
\hline T1: banano 70\%, arrocillo 30\%, cobertura de cera carnauba & $41.50 \pm 1.15$ \\
\hline T2: palma 70\%, arrocillo 30\%, cobertura de cera carnauba & $47.21 \pm 2.05$ \\
\hline T3: banano 70\%, arrocillo 30\%, cobertura de cera de abeja & $36.33 \pm 1.85$ \\
\hline T4: palma 70\%, arrocillo 30\%, cobertura de cera de abeja & $42.06 \pm 2.17$ \\
\hline Testigo: poliestireno expandido & $0.08 \mathrm{kN}$ \\
\hline
\end{tabular}

Fuente: Elaboración propia a partir de algunos datos tomados de Asociación Nacional de Poliestireno Expandido (2011). 
Tabla 5. Unidades Formadoras de Colonias (UFC) por tratamiento analizado.

\begin{tabular}{|l|l|}
\hline \multicolumn{1}{|c|}{ Tratamiento } & Colonias (UFC)-grupo Tukey \\
\hline T1: banano 70\%, arrocillo 30\%, cobertura de cera carnauba & $10 \mathrm{a}$ \\
\hline T2: palma 70\%, arrocillo 30\%, cobertura de cera carnauba & $0 \mathrm{a}$ \\
\hline T3: banano 70\%, arrocillo 30\%, cobertura de cera de abeja & $44 \mathrm{~b}$ \\
\hline T4: palma 70\%, arrocillo 30\%, cobertura de cera de abeja & $98 \mathrm{~b}$ \\
\hline Testigo: poliestireno expandido & $21 \mathrm{~b}$ \\
\hline
\end{tabular}

Fuente: Elaboración propia.

Los datos muestran claramente que existe diferencia entre el número de colonias que presentan los tratamientos en esta evaluación, donde el tratamiento 2 es el de menor probabilidad de contaminación frente al resto. Los tratamientos con cobertura de cera de abeja mantienen una contaminación más alta al momento de la valoración y el testigo también presenta colonización de mesófilos más alta que en los tratamientos con cobertura de cera carnauba.

La evaluación de microorganismos mesófilos aerobios a los 30 días de medición presentó una contaminación generalizada en todos los tratamientos, en donde se pudo comprobar que, para el día 30, los números de UFC son incontables. Este resultado se presentó en todos los tratamientos evaluados.

\section{Análisis microbiológico en periodos de 15 y 30 días de incubación}

Se consideró de importancia el análisis de mohos y levaduras en concordancia con que el material evaluado se encuentra en contacto con agua durante todo el tiempo de cultivo del vegetal. Del análisis estadístico se obtuvo un $p$-valor de 0.5 , siendo su resultado cualquier tratamiento y en el testigo.

\section{DISCUSIÓN}

Los prototipos desarrollados utilizaron metodologías artesanales simplificadas para su elaboración y alcanzaron los resultados funcionales esperados en cuanto a la flotabilidad durante todo el ciclo de cultivo, por tanto, su utilización es favorable como material de soporte en sistemas hidropónicos de raíz desnuda.

Al utilizar la metodología de elaboración de materiales descrita por Tamayo et al. (2017), se corroboró que la utilización de arrocillo como material ligante entre las fibras vegetales es eficiente con otras fibras, como sucedió con la palma aceitera (Elaeis guineensis).
Al ser un aglomerado de fibras vegetales con cobertura de polímeros orgánicos, las pruebas mecánicas superaron los valores presentados bibliográficamente del poliestireno expandido.

Es importante destacar que el aglomerado no fue desarrollado con pegamentos industriales como la urea-formaldehído, más bien, está constituido exclusivamente por materiales orgánicos biodegradables $\mathrm{y}$, pese a eso, los valores alcanzados en las pruebas mecánicas se encuentran dentro de los rangos presentados para los aglomerados comerciales de la Norma Técnica Ecuatoriana NTE INEN 896:2005. Tableros de madera aglomerada, contrachapada y de fibras de madera (MDF) (Instituto Ecuatoriano de Normalización, 1995; Instituto Ecuatoriano de Normalización, 2005).

Para la utilización del material en campo es necesario que la temperatura del sistema acuoso sobre el cual se desarrolle el cultivo mantenga un rango estable, ya que el incremento de temperatura puede afectar de manera importante la estabilidad de la cobertura polimérica del aglomerado.

Los resultados de las evaluaciones microbiológicas obtenidos en la medición a los 15 días de cultivo se encuentran por debajo del máximo permitido por la norma sanitaria (Ministerio de Salud del Perú, 2008), la cual indica un límite máximo de $10^{6}$ UFC.

\section{CONCLUSIONES}

Se concluye que los prototipos generados a partir de desechos agroindustriales de palma aceitera (Elaeis guineensis) y de banano (Musa paradisiaca), aglutinados con arrocillo y con cobertura de cera carnauba pueden cumplir las funciones del poliestireno expandido en cultivos de lechuga bajo el sistema de producción a raíz desnuda; por lo tanto, pueden reemplazarlo. Asimismo, se detectó que el material de mejores características fue el desarrollado con $70 \%$ de fibra de palma aceitera, $30 \%$ de 
arrocillo y con cobertura de cera carnauba. Por último, vale indicar que, al finalizar el ciclo de cultivo, el material puede ser enviado a sistemas de compostaje para su respectiva degradación y formación de abonos orgánicos.

\section{AGRADECIMIENTOS}

A la Universidad de Las Américas, a la Universidad Salesiana de Cuenca y a sus respectivos docentes, quienes colaboraron con la toma de datos de los análisis mecánicos y microbiológicos de los materiales desarrollados en esta investigación.

\section{REFERENCIAS BIBLIOGRÁFICAS}

[1] Asociación Nacional de Poliestireno Expandido (2011). Propiedades del EPS. Recuperado de http://www.anape.es/pdf/ficha73.pdf.

[2] Bejarano, G. (2006). Estudio de factibilidad para la producción de aceite de palmiste en Quinindé provincia de Esmeraldas. (Tesis de grado). Universidad San Francisco de Quito, Ecuador. Recuperado de http://repositorio. usfq.edu.ec/bitstream/23000/191/1/81021.pdf.

[3] Benavidez, R. A. (2006). El arroz. Su cultivo y sustentabilidad en Entre Ríos. Concepción del Uruguay, Argentina: Universidad Nacional de Entre Ríos / Universidad Nacional del Litoral.

[4] Cuá, A. y Vázquez, E. (2016). Manual de creación y manejo de hidroponía. San Antonio Suchitepéquez, Guatemala: Colegio Científico Tecnológico Einstein. Recuperado de Guzmán, G. (2004). Hidroponía en casa: una actividad familiar. Recuperado de http://usi.earth.ac.cr/ glas/sp/Hidroponia.pdf.

[5] HyServe (2010). Compact Dry EC Producto. HyServe. Instituto Ecuatoriano de Normalización (1995). Norma técnica ecuatoriana para tableros de partículas. Quito, Ecuador: República de Ecuador.

[6] Instituto Ecuatoriano de Normalización (2005). Norma técnica ecuatoriana NTE INEN 896:2005. Tableros de madera aglomerada, contrachapada y de fibras de madera (MDF). Determinación del contenido de humedad. Quito, Ecuador: República de Ecuador. Recuperado de https://archive.org/details/ ec.nte.0896.2005.
[7] López, S. y Ozaeta, G. (2013). Extracción de almidón a partir de arroz de rechazo molido como viscosante en la elaboración de cinco cosméticos. (Tesis de grado). Universidad de San Carlos de Guatemala, Guatemala.

[8] Manrique, A. y Rivera, D. (2012). Aprovechamiento de los residuos del pseudotallo del banano común (musa sp AAA) y del bocadillo (musa sp AA) para la extracción de fibras textiles. (Tesis de grado). Universidad Tecnológica de Pereira, Colombia. Recuperado de http://recursosbiblioteca.utp.edu.co/tesisd/ textoyanexos/6284458M285.pdf.

[9] Ministerio de Salud del Perú (2008) Norma sanitaria que establece los criterios microbiológicos de calidad sanitaria e inocuidad para los alimentos y bebidas de consumo humano. Recuperado de: https://www. saludarequipa.gob.pe/desa/archivos/Normas_ Legales/alimentos/RM591MINSANORMA.pdf.

[10] Rodríguez, I. (14 de setiembre de 2013). Biomaterial sustituto del poliestireno expandido. Saberes y Ciencias, (19). Recuperado de https://saberesyciencias.com.mx/2013/09/14/ biomaterial-sustituto-del-poliestirenoexpandido/.

[11] Tamayo, E., Sarasty, O. y Mosquera, E. (2017). Aprovechamiento de residuos ligno-celulósicos en la elaboración de empaques secundarios ecológicos. Industrial Data, 20(2), 37-42. Recuperado de https://revistasinvestigacion. unmsm.edu.pe/index.php/idata/article/ view/13959.

[12] Tapia, C. (2007). Elaboración de tableros de partícula fina a partir de residuos lignocelulósicos y resinas termoestables. (Tesis de grado). Escuela Superior Politécnica del Litoral, Ecuador. Recuperado de https:// www.academia.edu/1417600/Elaboración_ de_Tableros_de_Partícula_Fina_a_partir_ de_Residuos_Lignocelulósicos_y_Resinas_ Termoestables. 


\section{Evaluation of Biodegradable Floating Grow Mat Materials for Hydroponically Grown Lettuce (Lactuca sativa)}

Evelin TAMAYo GutiÉRreZ ${ }^{1}$ Bonye Carrera POZO ${ }^{2}$ MAURICIO RACINES-OLIVA ${ }^{3}$

ReCiBido: 20/05/2018 Aceptado: 04/11/2019 Publicado: 16/10/2020

\begin{abstract}
This study proposes an alternative biodegradable material for use with lettuce crops utilizing the bareroot hydroponic system, with the aim of functionally replacing expanded polystyrene, commonly used as a supporting material despite being a highly contaminating substance. This proposal is based on the production of individual pieces of floating material made from agroindustrial waste with an organic wax waterproof coating. The materials were produced in Quito, Ecuador, and the variables analyzed were hardness, compressive strength, flexural strength and microbiological contamination of the material during the vegetable growing period. Results showed that the alternative substrate with the greatest adaptability to the crops was made from a carnauba waxcoated palm fiber (Elaeis guineensis) blended with rice, in a ratio of $70 \%-30 \%$, respectively.
\end{abstract}

Keywords: floating grow mat materials; hydroponics; expanded polystyrene; palm fiber.

\section{INTRODUCTION}

Hydroponic cultivation of vegetables is an alternative to intensive agriculture, from which high volumes of production are obtained per square meter. It can be implemented in places where soil is unsuitable for agriculture, in reduced areas of land or in areas where water supply is limited, since water recirculation systems save up to $90 \%$ of water compared to vegetable cultivation in soil (Cuá \& Vázquez, 2016).

Hydroponic crops utilize materials in place of soil, and moreover provide a favorable environment for the plant, enabling its normal growth. In bare-root hydroponics, the plant is fixed on a grow mat floating on an aqueous nutrient solution during the whole growing cycle, until reaching the harvesting stage (Guzmán, 2004).

This research addresses the bare-root hydroponics system used for the cultivation of lettuce, where expanded polystyrene sheets are commonly used as base and grow mat for plants.

In spite of being a stable, low density, and easily accessible material used in this cultivation system, expanded polystyrene constitutes one of the most polluting materials after its useful life, due to its low biodegradability capacity (Rodríguez, September 14, 2013). This represents a high-impact environmental problem, upon which efforts to find an environmentally friendly material to replace it are being made (Tamayo, Sarasty \& Mosquera, 2017).

In light of the above, our proposal was aimed at producing biodegradable floating grow materials manufactured from agro-industrial waste as an alternative to expanded polystyrene that can fulfill plant support functionalities for bare-root hydroponically-grown lettuce.

$1 \mathrm{PhD}$ in Industrial Engineering from the Universidad Nacional Mayor de San Marcos (Lima, Peru). Currently working as professor of Agro-industrial and Food Engineering at the School of Engineering and Applied Sciences of the Universidad de Las Américas. (Quito, Ecuador). ORCID: https://orcid.org/0000-0003-3200-8229 E-mail: evelin.tamayo@udla.edu.ec

2 Agro-industrial and food engineer from the Universidad de Las Américas (Quito, Ecuador). Currently working as an independent consultant. (Quito, Ecuador). ORCID: https://orcid.org/0000-0002-5740-0748 E-mail: bmcarrera@udlanet.ec

$3 \mathrm{PhD}$ in Bioscience Engineering from the KU Leuven (Lovaina, Belgium). Research professor of Agro-industrial and Food Engineering at the Universidad de Las Américas. (Quito, Ecuador). ORCID: https://orcid.org/0000-0003-4335-4311 E-mail: mauricio.racines@udla.edu.ec 
Banana (Musa paradisiaca) agro-industrial waste was chosen as the primary input in the production of the plant fiber blend substrates because there are promising studies on its use at an industrial level, such as that of Manrique and Rivera (2012). Additionally, the behavior of banana fiber was compared to that of oil palm fiber (Elaeis guineensis). Finally, rice (Oryza sativa) was used as binding material due to its high starch content (López \& Ozaeta, 2013). For the input selection, it was taken into consideration that the crops mentioned above are of economic significance in Ecuador and constitute a supply of easily accessible, low cost raw material.

In the case of palm fiber, it is estimated that the amount of fiber obtained, after oil extraction, reaches $58.7 \%$ of its initial weight (Bejarano, 2006). Meanwhile, banana fiber extracted from the rachis constitutes between $12 \%$ and $15 \%$ of its total weight. Regarding rice, a byproduct called arrocillo was used, that is, broken rice produced during milling; this byproduct represents $10 \%$ of the total weight of rice to be milled (Benavidez, 2006).

Simplified unit operations were used to develop the prototypes with minimal energy consumption and without emission of polluting gases, so that this technology could be available to producers who wish to develop their own substrate materials.

In addition, it is important to emphasize that the use of byproducts and agro-industrial waste opens doors to new income opportunities, as it encourages the generation of value-added alternative products, that, if produced at industrial level, may create sources of employment (Tapia, 2007).

\section{METHODOLOGY}

\section{Materials and methods}

This study was conducted in the city of Quito, Ecuador, located at $2850 \mathrm{MASL}$, with an average annual temperature of $13.9^{\circ} \mathrm{C}$ and annual precipitation of $1273 \mathrm{~mm}$. The plant fiber blend substrate design described in the study by Tamayo et al. (2017) was used as the technical basis. In this context, oil palm (Elaeis guineensis) and banana (Musa paradisiaca) fibers were blended using INIAP15 broken rice, and then coated with organic bee and carnauba wax. Given the easy access of these materials, the formula employed was the one indicated in the abovementioned study, $70 \%$ banana fiber and $30 \%$ broken rice, seeing that it is a treatment with excellent results in terms of agglutination, compaction and homogeneity. Furthermore, the treatment was comparatively analyzed against plant fiber blend substrates made from oil palm fiber in equal concentrations.

Figure 1 shows the process diagram used for the development of the prototype.

The randomized complete block design (RCBD) was applied to the experiment, as well as the analysis of variance (ANOVA); Tukey's test $(p \leq 0.05)$ was also applied for comparison using SPSS software (International Business Machines Corporation, EE. UU.).

For the analysis, 25 plant fiber blend substrates of $12 \times 12 \times 3 \mathrm{~cm}$ were produced for each of the treatments described in Table 1.

Figure 2 shows the substrate material fabricated with plant fibers and an organic polymer coating, which was used in the study of hydroponic lettuce crops.

The prototypes were subjected to mechanical testing to determine surface hardness, compressive strength and flexural strength, as shown in Figure 3 (sections $A, B$ and $C$, respectively). In addition, the models were subjected to microbiological testing.

With respect to mechanical testing, each treatment was tested six times.

A durometer was used to measure surface hardness in Shore scale; both surfaces of the material were measured manually. To conduct the testing, a cross template was used in order to homologate the sampling in all the prototypes, resulting in a total of 32 penetrations per surface.

Specimens of $12 \times 4 \times 3 \mathrm{~cm}$ were prepared for flexural and compressive strength testing. Compressive strength was measured using a $3000 \mathrm{kN}$ compression equipment (see Figure 3 , section $B$ ); load capacity was measured in kN. Flexural strength was tested using a universal testing machine (see Figure 3 , section C) with a compressive capacity of 200 $\mathrm{kN}$, in order to determine the breaking load of each sample.

Microbiological testing was conducted with the premise that the developed material would be in direct contact with vegetables that are consumed raw, which is why this testing was considered important.

As shown in Figure 4, a cultivation system of individual units, with 6 samples each, was developed for the hydroponic cultivation of lettuce, where microbiological contamination of the material was tested. 


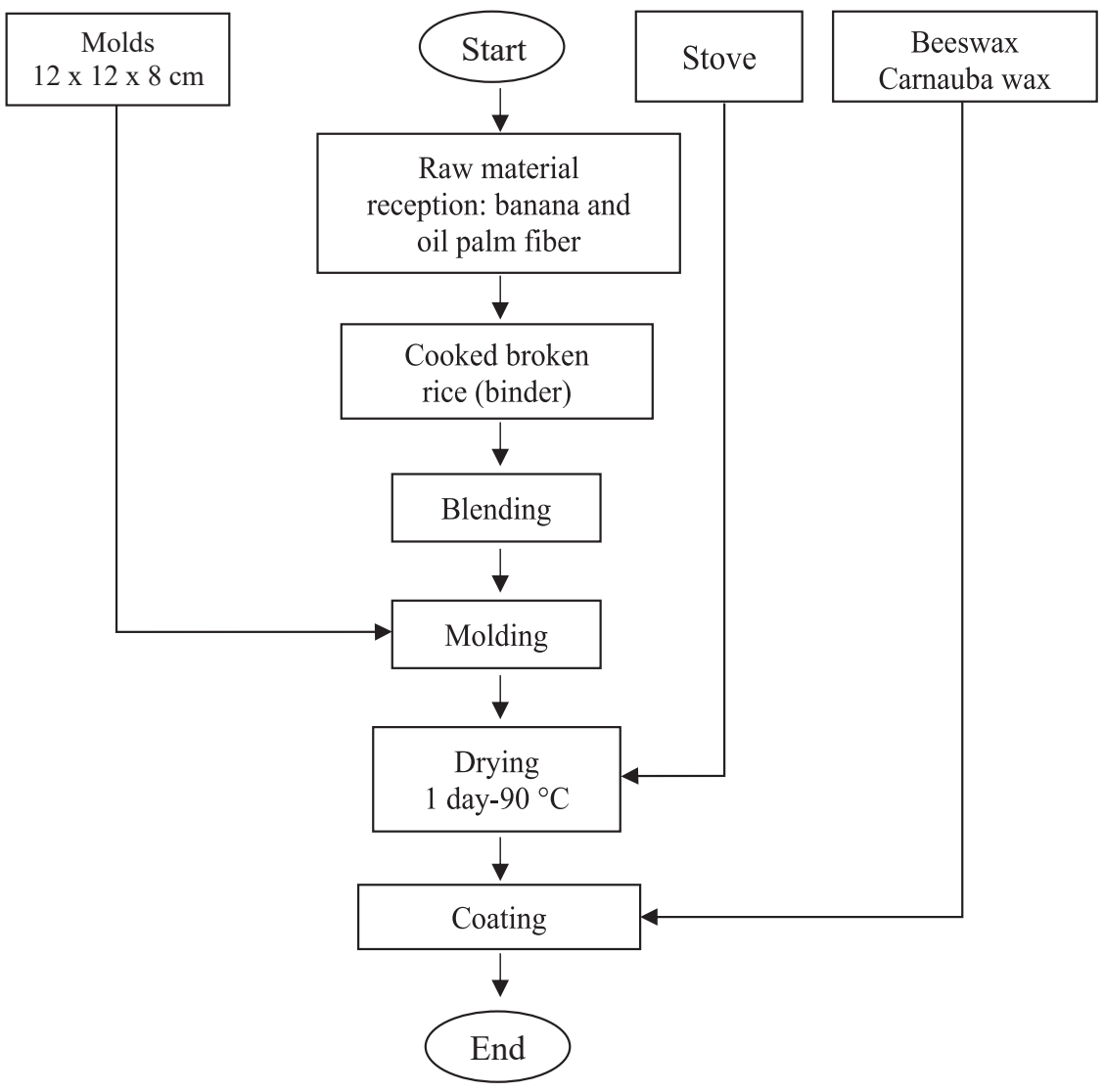

Figure 1. Process diagram for the elaboration of floating grow mat material prototypes. Source: Prepared by the authors.

Table 1. Description of treatments.

\begin{tabular}{|c|l|c|c|c|}
\hline Treatment & \multicolumn{1}{|c|}{ Fiber's origin } & $\begin{array}{c}\text { Fiber present in substrate } \\
\text { (\% dry weight) }\end{array}$ & $\begin{array}{c}\text { Broken rice present in substrate } \\
\text { (\% dry weight) }\end{array}$ & Coating \\
\hline 1 & Banana & $70 \%$ & $30 \%$ & Carnauba wax \\
\hline 2 & Oil palm & $60 \%$ & $40 \%$ & Carnauba wax \\
\hline 3 & Banana & $50 \%$ & $50 \%$ & Beeswax \\
\hline 4 & Oil palm & $70 \%$ & $30 \%$ & Beeswax \\
\hline 5 & Control: expanded polystyrene & & \\
\hline
\end{tabular}

Source: Prepared by the authors.

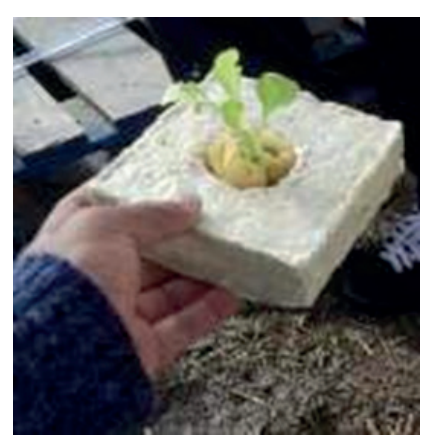

Figure 2. Prototype of plant fiber blend substrate with organic polymer coating. Source: Prepared by the authors. 
Samples were taken from the surface of each prototype under analysis following a zigzag pattern. Two key stages were considered for sampling: 15 and 30 days after seeding, the latter being the harvest time for lettuce. Compact Dry plates were used to test microbiological contamination, as these plates favor a uniform and standard culture of samples. Some of the characteristics of these plates are:

- Compact Dry TC is a medium containing agar that facilitates the identification of total aerobes using tetrazolium salt, which colors colonies red.
- Compact Dry YM enables the identification of mold and yeasts colonies. It contains the chromogenic substrate X-Phos, which colors colonies blue.

The plates used are certified by AOAC, MicroVal y NordVal (Hyserve, 2010).

The protocols used were as follows:

\section{Surface sampling and sample transportation methods}

- Use of latex gloves to prevent crosscontamination.

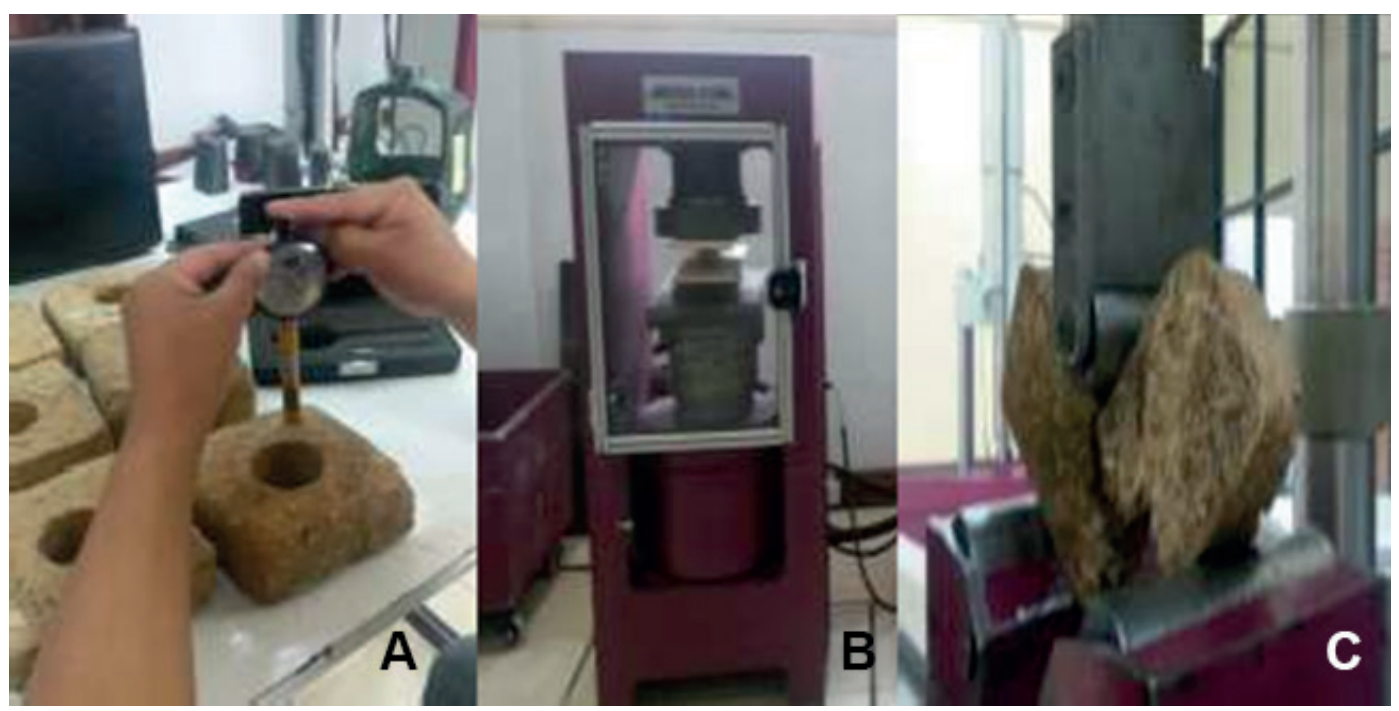

Figure 3. Mechanical testing. A: hardness measurement using durometer; B: compression strength measurement; $\mathrm{C}$ : flexure strength measurement.

Source: Prepared by the authors.

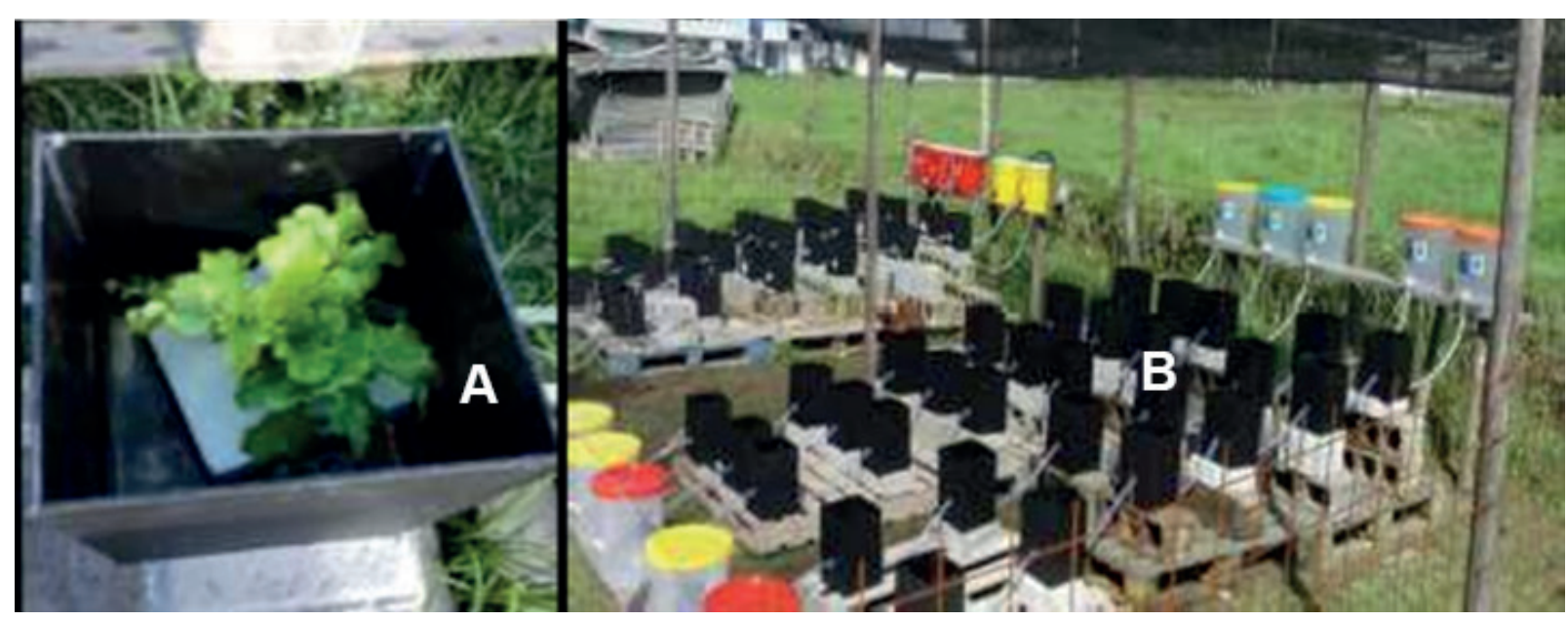

Figure 4. Cultivation system. A: testing of material used in independent units of the bare-root hydroponics system; $B$ : recirculation units for the testing of hydroponic lettuce crops.

Source: Prepared by the authors. 
- Random sampling.

- Scraping samples with a sterile cotton swabs following a zigzag pattern.

- Placement of the swabs in test tubes filled with distilled water to prevent any type of alteration on microorganisms.

- Transportation of test tubes transportation under safe conditions to prevent ruptures and temperature changes that could affect the sample.

\section{Sample streaking on cell-culture plates}

- The original sample was diluted in $10 \mathrm{ml}$ of distilled water and placed in test tubes.

- Then, it was diluted up to a $10^{-4}$ concentration.

- One milliliter of the described solution was placed onto the Compact Dry plates, labelling them according to treatment.

- Petri Compact Dry TC plates were incubated for 24 hours at a temperature range of 35 $37^{\circ} \mathrm{C}$.

- Culture plates were incubated for 48 to 72 hours at a temperature range of $25-30^{\circ} \mathrm{C}$.

Culture plates and colony count are shown in Figure 5.

\section{RESULTS}

The methodology used for the elaboration of prototypes from plant fibers blended with broken rice, described by Tamayo et al. (2017), generated homogeneous and stable materials, suitable for the hydroponic system studied.
Results of surface hardness testing showed significant difference between treatments; the prototype consisting of $70 \%$ oil palm, $30 \%$ broken rice and a carnauba wax coating proved to be the hardest material compared to its counterparts.

It is important to note that there is no statistical difference between treatments 1,3 , and 4. Data are exhibited in Table 2.

\section{Analysis of compressive strength testing results}

The analysis of the variance of breaking load rate yielded a $p$-value of less than 0.05 , thus there is no significant difference between the treatments; however, a difference was found between the treatments and the control group, where the latter had much lower compressive strength. Table 3 shows the averages (in $\mathrm{kN}$ ) of the treatments under study.

\section{Analysis of flexural strength testing results}

A $p$-value greater than 0.05 was obtained from the analysis, indicating that flexural strength values do not differ from treatment to treatment; thus, all prototypes have similar flexural strength before rupture, a result far superior than that of the control group. Table 4 shows the flexural strength averages (in $\mathrm{kN}$ ) obtained from the testing of prototypes.

\section{Microbiological analysis of aerobic mesophilic at 15 and 30 days}

The methodology described above was used to calculate the colony-forming unit (CFU) of aerobic mesophilic bacteria. Prototypes were analyzed after 15 days, and the results obtained are shown in Table 5.

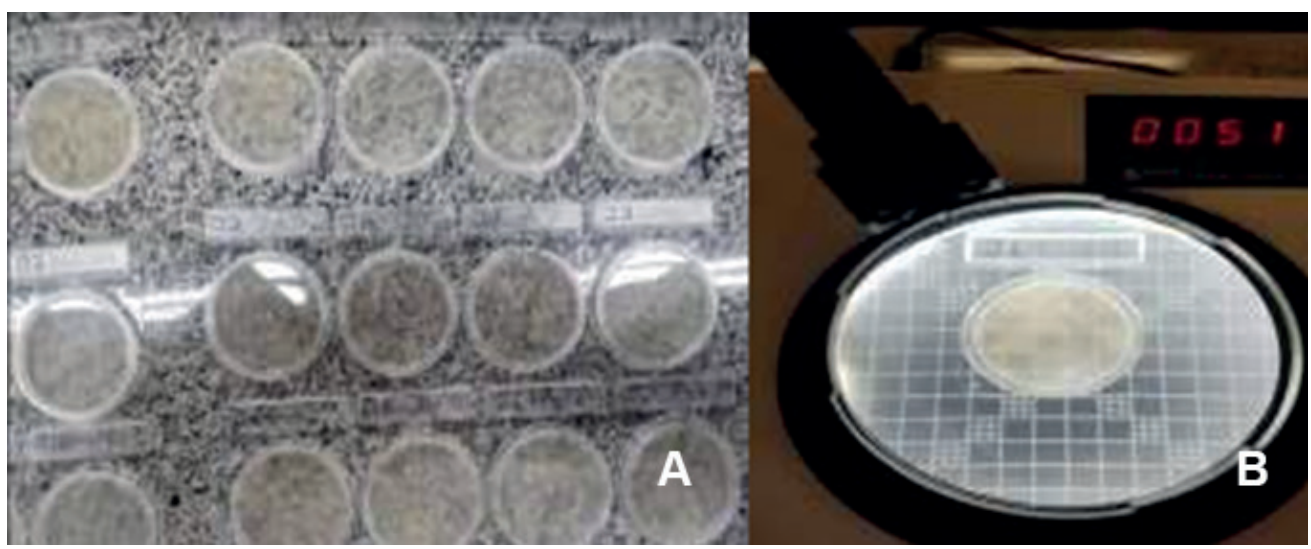

Figure 5. Microbiological testing. A: sample streaking using specific culture methods; B: colony count. Source: Prepared by the authors. 
The data clearly show that a difference exists between the number of colonies present in the treatments, where treatment 2 was the least likely to be contaminated compared to the rest. During the analysis, treatments with beeswax coating showed higher contamination values; the control also presented more aerobic mesophilic bacteria colonies than treatments with a carnauba wax coating.

The microbiological analysis conducted on samples taken 30 days after seeding found widespread aerobic mesophilic bacteria contamination in all treatments, which proved that the number or CFU are uncountable by day 30 . The same result was obtained in all treatments.

\section{Microbiological analysis conducted after 15 and 30-day incubation periods}

The analysis of molds and yeasts was considered important, as the material analyzed was in contact with water during the entire growing period of the plant. A $p$-value of 0.5 was obtained from the statistical analysis; therefore, there is no significant difference between the treatments, including the control group.

\section{DISCUSSION}

The prototypes developed utilized simplified artisanal methodologies for their production and achieved expected functional results throughout the growing cycle in terms of buoyancy; therefore, their use as supporting material in bare-root hydroponic systems is favorable.

By applying the methodology proposed by Tamayo et al. (2017) for the production of plant fiber blend substrates, the use of broken rice as a binding material was proven efficient with other plant fibers, as was the case with oil palm (Elaeis guineensis).

Being a plant fiber blend substrate with an organic polymer coating, the mechanical tests exceeded the reference values for expanded polystyrene.

It is important to note that no industrial glue such as urea-formaldehyde was used in the fabrication

Table 2. Significance analysis results on surface hardness testing obtained using Tukey's test.

\begin{tabular}{|l|c|c|}
\hline \multicolumn{1}{|c|}{ Treatment } & $\boldsymbol{\sigma} \pm \mathbf{S}^{2}$ & Significance group \\
\hline T1: $70 \%$ banana, 30\% broken rice, carnauba wax coating & $17.87 \pm 0.35$ & $\mathrm{~b}$ \\
\hline T2: $70 \%$ oil palm, 30\% broken rice, carnauba wax coating & $23.12 \pm 0.16$ & $\mathrm{c}$ \\
\hline T3: $70 \%$ banana, 30\% broken rice, beeswax coating & $15.90 \pm 0.86$ & $\mathrm{~b}$ \\
\hline T4: $70 \%$ oil palm, 30\% broken rice, beeswax coating & $16.73 \pm 0.11$ & $\mathrm{~b}$ \\
\hline
\end{tabular}

Source: Prepared by the authors.

Table 3. Analysis of compressive strength testing results using Tukey's test.

\begin{tabular}{|l|l|}
\hline \multicolumn{1}{|c|}{ Treatment } & \multicolumn{1}{c|}{ Average (kN) } \\
\hline T1: 70\% banana, 30\% broken rice, carnauba wax coating & $364.50 \pm 12.15$ \\
\hline T2: 70\% oil palm, 30\% broken rice, carnauba wax coating & $374.00 \pm 10.05$ \\
\hline T3: 70\% banana, 30\% broken rice, beeswax coating & $432.33 \pm 18.85$ \\
\hline T4: 70\% oil palm, 30\% broken rice, beeswax coating & $420.67 \pm 14.17$ \\
\hline Control: expanded polystyrene & $245 \mathrm{kN}$ \\
\hline
\end{tabular}

Source: Prepared by the authors based on data from the Asociación Nacional de Poliestireno Expandido (2011).

Table 4. Analysis of flexural strength testing results using Tukey's test.

\begin{tabular}{|l|l|}
\hline \multicolumn{1}{|c|}{ Treatment } & \multicolumn{1}{c|}{ Average (kN) } \\
\hline T1: 70\% banana, 30\% broken rice, carnauba wax coating & $41.50 \pm 1.15$ \\
\hline T2: 70\% oil palm, 30\% broken rice, carnauba wax coating & $47.21 \pm 2.05$ \\
\hline T3: 70\% banana, 30\% broken rice, beeswax coating & $36.33 \pm 1.85$ \\
\hline T4: 70\% oil palm, 30\% broken rice, beeswax coating & $42.06 \pm 2.17$ \\
\hline Control: expanded polystyrene & $0.08 \mathrm{kN}$ \\
\hline
\end{tabular}

Source: Prepared by the authors based on data from the Asociación Nacional de Poliestireno Expandido (2011). 
Table 5. Colony-forming units per treatment.

\begin{tabular}{|l|l|}
\hline Treatment & Colonies (CFU)-Tukey group \\
\hline T1: 70\% banana, 30\% broken rice, carnauba wax coating & $10 \mathrm{a}$ \\
\hline T2: 70\% oil palm, 30\% broken rice, carnauba wax coating & $0 \mathrm{a}$ \\
\hline T3: $70 \%$ banana, 30\% broken rice, beeswax coating & $44 \mathrm{~b}$ \\
\hline T4: 70\% oil palm, 30\% broken rice, beeswax coating & $98 \mathrm{~b}$ \\
\hline Control: expanded polystyrene & $21 \mathrm{~b}$ \\
\hline
\end{tabular}

Source: Prepared by the authors.

of the plant fiber blend substrates; on the contrary, it was made exclusively from biodegradable organic materials. In spite of that, the values reached in the mechanical tests were within the ranges for commercial particle boards established by the Norma Técnica Ecuatoriana NTE INEN 896:2005, Tableros de madera aglomerada, contrachapada y de fibras de madera (MDF), Ecuadorian standard that regulates engineered wood standards (Instituto Ecuatoriano de Normalización, 1995; Instituto Ecuatoriano de Normalización, 2005).

To use this material, temperature in the aqueous system must remain stable, as an increase in temperature can significantly affect the stability of the plant fiber blend substrate's polymer coating.

The results of microbiological testing obtained in the measurement conducted 15 days after seeding were below the maximum allowed by Peruvian health standards (Ministerio de Salud del Perú, 2008), which indicates a maximum limit of 106 CFU.

\section{CONCLUSIONS}

It is concluded that the prototypes made from oil palm (Elaeis guineensis) and banana (Musa paradisiaca) agro-industrial waste, blended with broken rice, and coated with carnauba wax can successfully perform the functions of expanded polystyrene in lettuce crops using the bare-root cultivation system; therefore, they can replace it. In addition, it was determined that the material fabricated using $70 \%$ oil palm fiber and $30 \%$ rice, with a carnauba wax coating, had the best characteristics. Finally, it should be noted that the material can be sent to composting plants for its decomposition and subsequent composting at the end of the growing cycle.

\section{ACKNOWLEDGMENT}

To the Universidad de Las Américas, Universidad Salesiana of Cuenca and to their faculty, who assisted us with the data collection of the mechanical and microbiological analyses conducted on the materials developed in this research.

\section{REFERENCES}

[1] Asociación Nacional de Poliestireno Expandido (2011). Propiedades del EPS. Retrieved from http://www.anape.es/pdf/ficha73.pdf.

[2] Bejarano, G. (2006). Estudio de factibilidad para la producción de aceite de palmiste en Quinindé provincia de Esmeraldas. (Degree thesis). Universidad San Francisco de Quito, Ecuador. Retrieved from http://repositorio.usfq. edu.ec/bitstream/23000/191/1/81021.pdf.

[3] Benavidez, R. A. (2006). El arroz. Su cultivo y sustentabilidad en Entre Ríos. Concepción del Uruguay, Argentina: Universidad Nacional de Entre Ríos / Universidad Nacional del Litoral.

[4] Cuá, A. \& Vázquez, E. (2016). Manual de creación y manejo de hidroponía. San Antonio Suchitepéquez, Guatemala: Colegio Científico Tecnológico Einstein. Retrieved from om/ read/000676235563a5afe3a26.

[5] Guzmán, G. (2004). Hidroponía en casa: una actividad familiar. Retrieved from http://usi. earth.ac.cr/glas/sp/Hidroponia.pdf.

[6] HyServe (2010). Compact Dry EC Producto. HyServe. Instituto Ecuatoriano de Normalización (1995). Norma técnica ecuatoriana para tableros de partículas. Quito, Ecuador: Republic of Ecuador.

[7] Instituto Ecuatoriano de Normalización (2005). Norma técnica ecuatoriana NTE INEN 896:2005. Tableros de madera aglomerada, contrachapada y de fibras de madera (MDF). Determinación del contenido de humedad. Quito, Ecuador: República de Ecuador. Retrieved from https://archive.org/details/ ec.nte.0896.2005.

[8] López, S. \& Ozaeta, G. (2013). Extracción de almidón a partir de arroz de rechazo molido 
como viscosante en la elaboración de cinco cosméticos. (Degree thesis). Universidad de San Carlos de Guatemala, Guatemala.

[9] Manrique, A. \& Rivera, D. (2012). Aprovechamiento de los residuos del pseudotallo del banano común (musa sp AAA) y del bocadillo (musa sp AA) para la extracción de fibras textiles. (Degree thesis). Universidad Tecnológica de Pereira, Colombia. Retrieved from http://recursosbiblioteca.utp.edu.co/ tesisd/textoyanexos/6284458M285.pdf.

[10] Ministerio de Salud del Perú (2008) Norma sanitaria que establece los criterios microbiológicos de calidad sanitaria e inocuidad para los alimentos y bebidas de consumo humano. Retrieved from: https://www. saludarequipa.gob.pe/desa/archivos/Normas Legales/alimentos/RM591MINSANORMA.pdf.

[11] Rodríguez, I. (September 14, 2013). Biomaterial sustituto del poliestireno expandido. Saberes y Ciencias, (19). Retrieved from https:// saberesyciencias.com.mx/2013/09/14/ biomaterial-sustituto-del-poliestireno-expandido/.
[12] Tamayo, E., Sarasty, O. \& Mosquera, E. (2017). Aprovechamiento de residuos ligno-celulósicos en la elaboración de empaques secundarios ecológicos. Industrial Data, 20(2), 37-42. Retrieved from https://revistasinvestigacion. unmsm.edu.pe/index.php/idata/article/ view/13959.

[13] Tapia, C. (2007). Elaboración de tableros de partícula fina a partir de residuos lignocelulósicos y resinas termoestables. (Degree thesis). Escuela Superior Politécnica del Litoral, Ecuador. Retrieved from https:// www.academia.edu/1417600/Elaboración_ de_Tableros_de_Partícula_Fina_a_partir_ de_Residuos_Lignocelulósicos_y_Resinas_ Termoestables. 